TAIWANESE JOURNAL OF MATHEMATICS

Vol. 14, No. 4, pp. 1609-1621, August 2010

This paper is available online at http://www.tjm.nsysu.edu.tw/

\title{
ORBIT SHIFTED SHADOWING PROPERTY OF GENERALIZED LOZI MAPS
}

\begin{abstract}
Akio Sakurai
Abstract. In this paper, we will show that certain Lozi-like maps have the orbit-shifted shadowing property.
\end{abstract}

\section{INTRODUCTION}

It is well known that pictures of strange attractors drawn by computer simulations are not necessarily similar to their actual figures. Indeed, rounding errors in computing are inevitable as long as we use existing computer machines with finite precision. So, we would like to know sufficient conditions which ensure that pseudo-orbits of a homeomorphism $f$ can be traceable by actual orbits even if such errors are not inevitable. This property is called the shadowing property of $f$. According to Anosov [1] and Bowen [2], uniformly hyperbolic diffeomorphisms have the shadowing property. However, if we relax the uniform hyperbolicity condition on diffeomorphisms slightly, then many problems concerning the shadowing property are not solved yet.

In this paper, we study some shadowing property of certain piecewise hyperbolic maps introduced by Young [11], which are generalized from two-parameter family of planar maps presented by Lozi [8]:

$$
L_{a, b}(x, y)=(1-a|x|+y, b x) .
$$

Note that $L_{a, b}$ is not differentiable on the $y$-axis. In general, such a nonempty subset where a given map is not differentiable is called a singularity set. Moreover, Kiriki studied forward limit sets of singularities for the Lozi famaily in [4]. According to [9] (see also [7]), the Lozi map $L_{a, b}$ admits a strange attractor $\Lambda_{a, b}$ in a bounded

Received October 20, 2008, accepted December 24, 2008.

Communicated by Jong-shenq Guo.

2000 Mathematics Subject Classification: 37D50, 37D45, 37C50.

Key words and phrases: Shadowing property, Lozi-like maps, Piecewise hyperbolic. 
region of $\mathbb{R}^{2}$ if the parameter $(a, b)$ belongs to some open set of the parameter space which is called the Misiurewicz domain.

Coven, Kan and Yorke [3] introduced the notion of the parameter-shifted shadowing property (for short PSSP). In fact, they proved that the tent maps $T_{a}(x)=$ $1-a|x|$ have the parameter-fixed shadowing property for almost every sloop $a$ in the open interval $I=(\sqrt{2}, 2)$, but do not have it for any $a$ in a certain dense subset of $I$. Moreover, they proved that, for any $a \in I$, the tent map $T_{a}$ has PSSP. This property was studied by Nusse and Yorke [10] by using the kneading theory. Kiriki and Soma proved that certain Lozi maps and geometric Lorenz maps have PSSP in [5] and [6] respectively.

In this paper, we study a certain of shadowing property in which we shift some entries of pseudo-orbits by rule instead of shifting parameters of maps, called the orbit-shifted shadowing property (for short OSSP). See Definition 2.3. We will show that certain piecewise Lozi-like maps (called generalized Lozi maps) introduced by Young [11] have the OSSP. In [11], it is proved that such maps have attractors with SRB-measure.

\section{Preliminaries}

First we present the definition of generalized Lozi maps according to [11]. Let $R=[0,1] \times[0,1]$ and let $f: R \rightarrow R$ be a continuous injective map. Suppose that $f$ (or some iterate of $f$ ) takes $R$ into its interior.

Definition 2.1. A continuous injective map $f: R \rightarrow R$ of $R=[0,1] \times[0,1]$ is a generalized Lozi map if it satisfies the following conditions.

(L.1) There exist $0<a_{1}<\cdots<a_{n}<1$ such that $f$ is a $C^{1}$-diffeomorphism on $R \backslash \bigcup_{i=1}^{n} Y_{i}$, where $Y_{i}=\left\{a_{i}\right\} \times[0,1]$. From now on, we set $S=\bigcup_{i=1}^{n} Y_{i}$.

(L.2) The norm of the derivative $D f$ of $f$ is uniformly bounded on $R \backslash S$, i.e.

$$
M_{f}=\sup \left\{\left\|D f_{\mathbf{x}}\right\| ; \mathbf{x} \in R \backslash S\right\}<\infty,
$$

where $\left\|D f_{\mathbf{x}}\right\|=\sup \left\{\left\|D f_{\mathbf{x}}(\mathbf{v})\right\| ; \mathbf{v} \in T_{\mathbf{x}}(R),\|\mathbf{v}\|=1\right\}$.

(L.3) There exist constants $\left|\lambda^{s}\right|<1<\left|\lambda^{u}\right|$ and continuous cone-fields $C^{s}=$ $\left\{C_{\mathbf{x}}^{s}\right\}_{\mathbf{x} \in R}, C^{u}=\left\{C_{\mathbf{x}}^{u}\right\}_{\mathbf{x} \in R}$ on $R$ such that, for any $\mathbf{x} \in R \backslash S$ and any vectors $\mathbf{v} \in C_{\mathbf{x}}^{u}, \mathbf{w} \in C_{f(\mathbf{x})}^{s}$,

$$
\text { - } D f_{\mathbf{x}}\left(C_{\mathbf{x}}^{u}\right) \subset C_{f(\mathbf{x})}^{u} \text { and }\left\|D f_{\mathbf{x}}(\mathbf{v})\right\| \geq\left|\lambda^{u}\right|\|\mathbf{v}\| \text {. }
$$$$
\text { - } D f_{f(\mathbf{x})}^{-1}\left(C_{f(\mathbf{x})}^{s}\right) \subset C_{\mathbf{x}}^{s} \text { and }\left\|D f_{f(\mathbf{x})}^{-1}(\mathbf{w})\right\| \geq\left|\left(\lambda^{s}\right)^{-1}\right|\|\mathbf{w}\| \text {. }
$$

We say that $C^{s}, C^{u}$ are stable and unstable cone-fields of $f$ respectively. 
Figure 2.1 illustrates the image $f(R)$ of $R$ by a generalized Lozi map $f$.

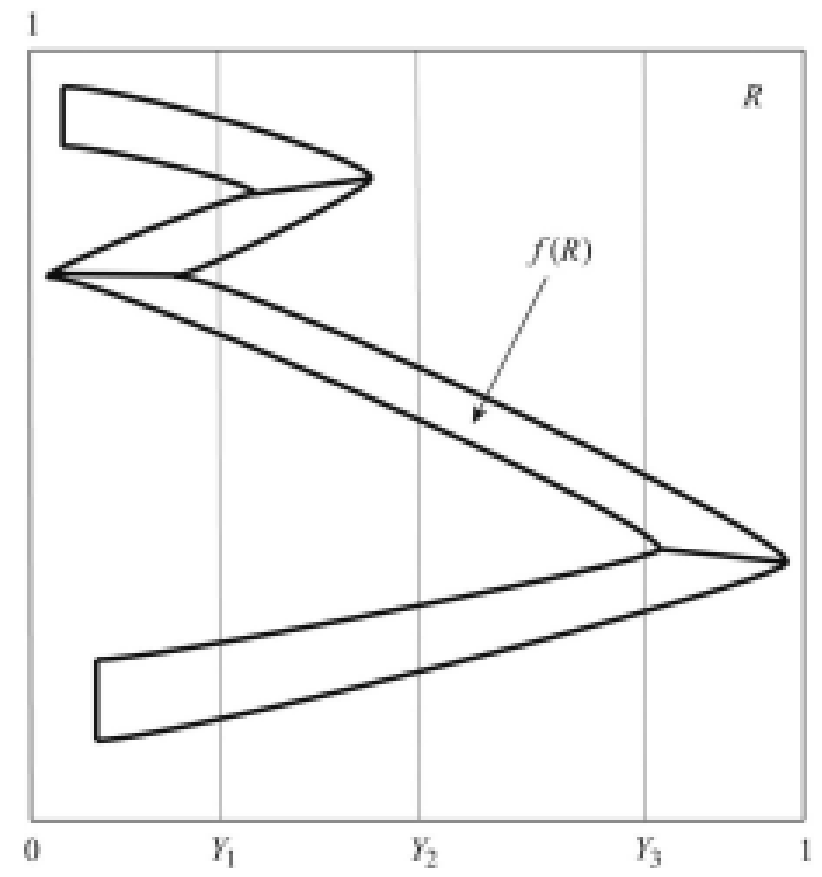

Figure 2.1.

In this paper, we consider generalized Lozi maps $f$ satisfying the following extra conditions (2.1)-(2.4).

$$
0<\lambda^{s} \leq \frac{1}{8} \quad \text { and } \quad \lambda^{u} \geq \sqrt{3}
$$

$$
C_{\mathbf{x}}^{s}=\left\{(u, v) \in T_{\mathbf{x}} R ;|v| \geq \alpha_{1}|u|\right\}, C_{\mathbf{x}}^{u}=\left\{(u, v) \in T_{\mathbf{x}} R ;|v| \leq \alpha_{2}|u|\right\},
$$

where $\alpha_{1}, \alpha_{2}$ are constants with

$$
\begin{gathered}
\alpha_{1} \geq \sqrt{3}, \quad 0<\alpha_{2} \leq \frac{1}{144} . \\
S \cap\left(f(S) \cup f^{2}(S)\right)=\emptyset .
\end{gathered}
$$

We note that any original Lozi maps $L_{a, b}$ in $[5, \S 1$, Theorem] satisfies the conditions (2.1)-(2.4).

An unstable curve $l$ in $R$ is a piecewise $C^{1}$-curve meeting $S$ transversely at most one point and satisfying $T_{\mathbf{x}} l \subset C_{\mathbf{x}}^{u}$ for any smooth point $\mathrm{x}$ of $l$. For any component 
$Y_{i}$ of $S$, consider an unstable curve $l$ in $R$ meeting $Y_{i}$ at an interior point $a(l)$ of $l$ and satisfying $f(l) \cap S=\emptyset$. Let $\varphi: l \rightarrow \mathbb{R}$ be the map defined by $\varphi(\mathbf{x})=\pi \circ f^{2}(\mathbf{x})$ for any $\mathbf{x} \in l$, where $\pi: \mathbb{R}^{2} \rightarrow \mathbb{R}$ is the orthogonal projection onto the $x$-axis, i.e. $\pi(x, y)=x$. Then we have the following three cases, see Figure 2.2.

(i) $\varphi(a(l))$ is a unique maximal point of $\varphi$.

(ii) $\varphi(a(l))$ is a unique minimal point of $\varphi$.

(iii) $\varphi(a(l))$ is an interior point of the interval $\varphi(l)$.

From the continuity of $D f_{\mathbf{x}}$ on $R \backslash S$, this property depends only on $Y_{i}$ and is independent of the choice of an unstable curve $l$ as above. The component $Y_{i}$ of $S$ is said to be of type 1 (resp. 2,3) if $\varphi$ satisfies the condition (i) (resp. (ii), (iii)). The union of components of $S$ of type $k$ is denoted by $S_{k}$ for $k=1,2,3$.

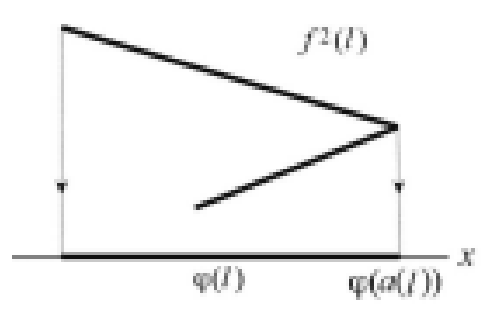

(i)

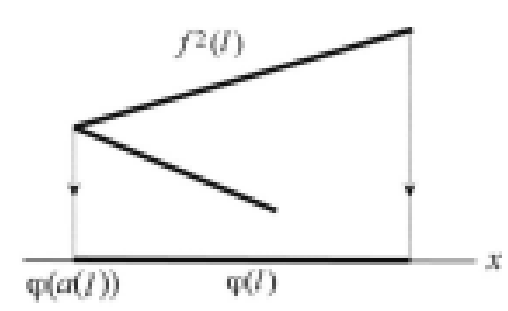

(ii)

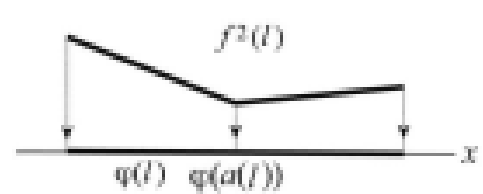

(iii)

Figure 2.2.

We say that the union $S_{1} \cup S_{2}$ is the essential singularity set of $f$.

Note that one can use $\pi \circ f$ instead of $\varphi=\pi \circ f^{2}$ to define types of $Y_{i}$. However, the replacement of types would complicate our arguments in Subsection 3.2.

By (L.2) and (2.4), there exists $\eta_{0}>0$ such that $B_{\eta_{0}}(\mathbf{x})$ meets at most one component of $S$ for any $\mathrm{x} \in R$ and

$$
f\left(B_{\eta_{0}}(\mathbf{x})\right) \cap S=\emptyset \quad \text { and } \quad f^{2}\left(B_{\eta_{0}}(\mathbf{x})\right) \cap S=\emptyset
$$

whenever $B_{\eta_{0}}(\mathbf{x}) \cap S \neq \emptyset$, where $B_{\eta_{0}}(\mathbf{x})$ is the closed disk in $R$ centered at $\mathbf{x}$ of radius $\eta_{0}$.

Definition 2.2. For $\delta_{0}>0, \delta_{1}>0$ and $\delta>0$ with $\delta_{0} \leq \eta_{0}$, a sequence $\left\{\mathbf{x}_{n}\right\}_{n \geq 0}$ in $R$ is an $\left(\delta_{0}, \delta_{1}\right)$-shifted $\delta$-pseudo-orbit of $f$ if, for any $n \geq 1, \mathbf{x}_{n}$ and $\mathbf{x}_{n+1}$ satisfy the following conditions. 
(i) $\left\|f\left(\mathbf{x}_{n}\right)-\mathbf{x}_{n+1}\right\| \leq \delta$ if $B_{\delta_{0}}\left(\mathbf{x}_{n-1}\right) \cap\left(S_{1} \cup S_{2}\right)=\emptyset$.

(ii) $\left\|f\left(\mathbf{x}_{n}\right)-\left(\delta_{1}, 0\right)-\mathbf{x}_{n+1}\right\| \leq \delta$ if $B_{\delta_{0}}\left(\mathbf{x}_{n-1}\right) \cap S_{1} \neq \emptyset$.

(iii) $\left\|f\left(\mathbf{x}_{n}\right)+\left(\delta_{1}, 0\right)-\mathbf{x}_{n+1}\right\| \leq \delta$ if $B_{\delta_{0}}\left(\mathbf{x}_{n-1}\right) \cap S_{2} \neq \emptyset$.

Figure 2.3 illustrates an example of a $\left(\delta_{0}, \delta_{1}\right)$-shifted $\delta$-pseudo-orbit of $f$ in the case (ii).

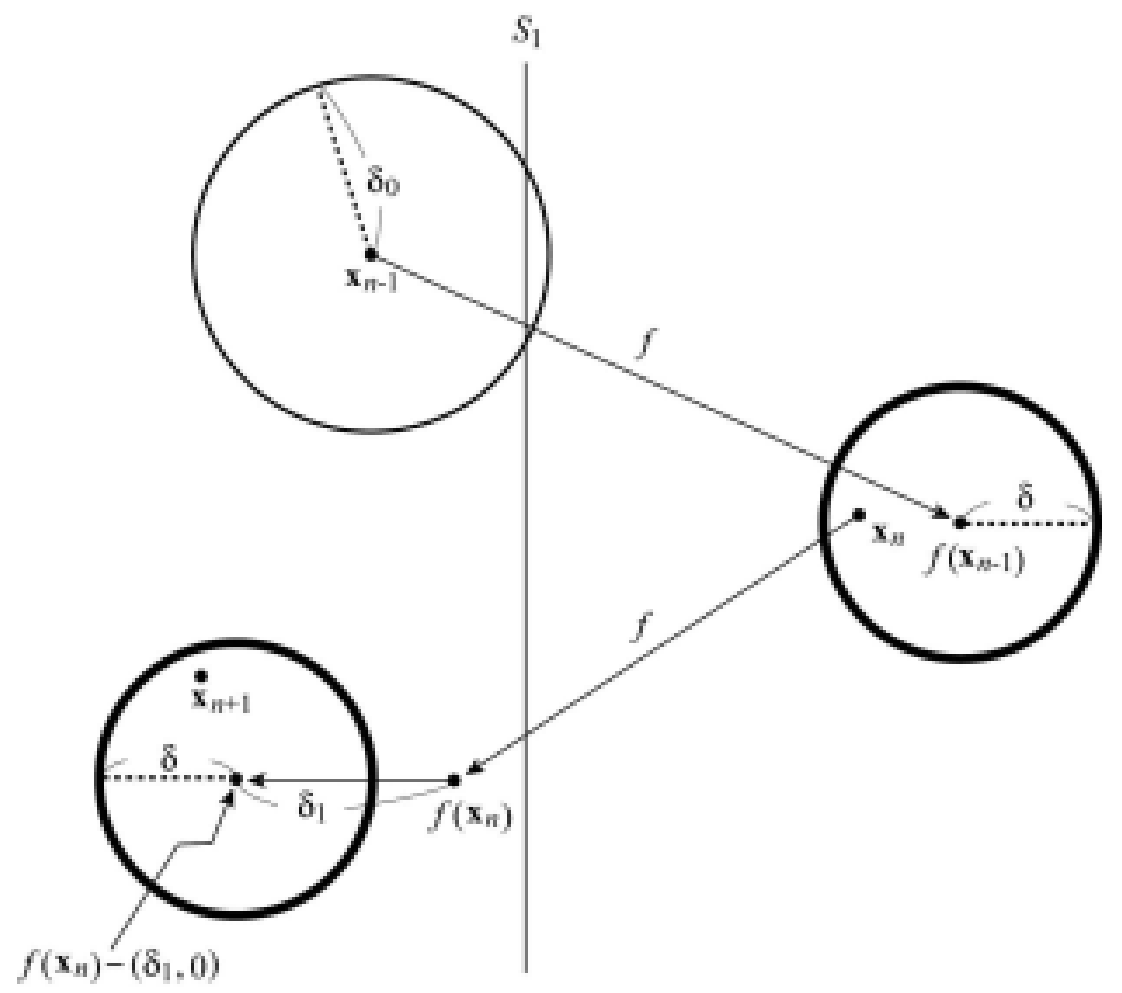

Figure 2.3.

Definition 2.3. We say that $f$ has the orbit-shifted shadowing property (for short $O S S P$ ) if, for any $\varepsilon>0$ with $\varepsilon \leq \eta_{0}$, there exist $\delta_{0}, \delta_{1}, \delta>0$ such that any $\left(\delta_{0}, \delta_{1}\right)$-shifted $\delta$-pseudo-orbit $\left\{\mathbf{x}_{n}\right\}_{n \geq 0}$ in $R$ of $f$ can be $\varepsilon$-shadowed by an actual orbit of $f$, that is, there exists $\mathbf{z} \in R$ such that $\left\|f^{n}(\mathbf{z})-\mathbf{x}_{n}\right\| \leq \varepsilon$ for any $n \geq 0$.

Recall that we fix a original map once and for all and use a certain of pseudoorbit satisfying the condition in Definition 2.2 instead of shifting parameters of maps in PSSP.

The following is our main result. 
Theorem 2.4. Any generalized Lozi map $f$ satisfying the conditions (2.1)-(2.4) has the orbit-shifted shadowing property. More precisely, for any $0<\varepsilon \leq \eta_{0}$, there exists $\delta>0$ such that any $(\varepsilon, \varepsilon / 2)$-shifted $\delta$-pseudo-orbit of $f$ in $R$ is $\varepsilon$-shadowed by an actual orbit.

\section{Proof of the TheOREM}

In this section we prove our main theorem.

Let $l$ be any piecewise $C^{1}$-curve $l$ in $R$ and $\partial l$ the end point set of $l$. The length of $l$ is denoted by $|l|$ and the subarc of $l$ with end point set $\{\mathbf{x}, \mathbf{y}\}$ by $l_{[\mathbf{x}, \mathbf{y}]}$.

Throughout the remainder of this paper, fix a non-singular vector field $X$ on $R \backslash\left(S \cup f^{-1}(S)\right)$ with $X(\mathbf{x}) \in D f_{f^{2}(\mathbf{x})}^{-2}\left(C_{f^{2}(\mathbf{x})}^{s}\right)$ for any $\mathbf{x} \in R \backslash\left(S \cup f^{-1}(S)\right)$ which is continuous on each component of $R \backslash\left(S \cup f^{-1}(S)\right)$. Note that $X$ is not necessarily extended to a continuous vector field on $R$. A piecewise $C^{1}$-curve $\gamma$ in $R$ meeting $S \cup f^{-1}(S)$ in at most finitely many points is called an integral curve with respect to $X$ if $\gamma$ is tangent to $X$ at any point of $\gamma \backslash\left(S \cup f^{-1}(S)\right)$. By (2.1), we have, for any integral curve $\gamma$,

$$
|f(\gamma)| \leq \frac{1}{8}|\gamma| \quad \text { and } \quad\left|f^{2}(\gamma)\right| \leq \frac{1}{64}|\gamma| .
$$

We say that a point $\mathbf{w}$ of an unstable curve $l$ is the foot of $\mathbf{x} \in R$ in $l$ if there exists an integral curve with respect to $X$ connecting $\mathbf{x}$ with $\mathbf{w}$.

For any $0<\varepsilon \leq \eta_{0}$, we put

$$
\begin{aligned}
a & :=\frac{\varepsilon}{36}, \\
\delta & :=\frac{a}{8 M_{f}}=\frac{\varepsilon}{288 M_{f}},
\end{aligned}
$$

where $M_{f}$ is the constant given in (L.2) of Definition 2.1. Since $M_{f} \geq \sqrt{3}$,

$$
\delta \leq \frac{a}{8}=\frac{\varepsilon}{288} .
$$

Lemma 3.1. Let $l$ be an unstable curve in $R$ with $|l|=\varepsilon$ and $\gamma$ an integral curve with respect to $X$ which connects a point $\mathbf{x}$ in $R$ and a point $\mathbf{w}$ in $l$. If there exits a point $\mathbf{v}$ in $l$ with $\|\mathbf{v}-\mathbf{x}\| \leq a$, then $|\gamma|<2 a$ and $\left|l_{[\mathbf{w}, \mathbf{v}]}\right|<2 a$. In particular, $\|\mathbf{x}-\mathbf{w}\|<2 a$.

Proof. Set $\partial l=\left\{\mathbf{u}_{0}, \mathbf{u}_{1}\right\}, \mathbf{u}_{0}=\left(a_{0}, b_{0}\right)$ and $\mathbf{u}_{1}=\left(a_{1}, b_{1}\right)$. Let $\Delta$ be the isosceles triangle as in Figure 3.1 satisfying the following conditions: 
- The two sides of $\Delta$ meeting at $\mathbf{u}_{0}$ are contained in lines given by $y=$ $\pm \frac{1}{144}\left(x-a_{0}\right)+b_{0}$, respectively.

- The vertex of $\Delta$ is located at $\mathbf{u}_{0}=\left(a_{0}, b_{0}\right)$ and the base $B$ of $\Delta$ belongs to the line $x=b_{1}$.

Since $l$ is an unstable curve, by (2.2) and (2.3), $l$ is contained in $\Delta$. Let $L$ be the line in $\mathbb{R}^{2}$ passing through one of the end points of $B$ and parallel to the $x$-axis and $p: \mathbb{R}^{2} \rightarrow L$ the orthogonal projection. One can choose $L$ so that the integral curve $\gamma^{\prime}$ connecting $\mathbf{x}$ and its foot in $L$ includes $\gamma$. Set $p(\mathbf{x})=\mathbf{x}^{\prime}, p(\mathbf{v})=\mathbf{v}^{\prime}$ and $p(\mathbf{w})=\mathbf{w}^{\prime}$.

Since $|l|=\varepsilon$, it follows that $|B| \leq 2 \varepsilon / 144=\varepsilon / 72$ and hence

$$
\left\|\mathbf{x}-\mathbf{x}^{\prime}\right\| \leq\|\mathbf{x}-\mathbf{v}\|+\left\|\mathbf{v}-\mathbf{v}^{\prime}\right\|<a+\frac{\varepsilon}{72}=a+\frac{36}{72} a=\frac{3}{2} a .
$$

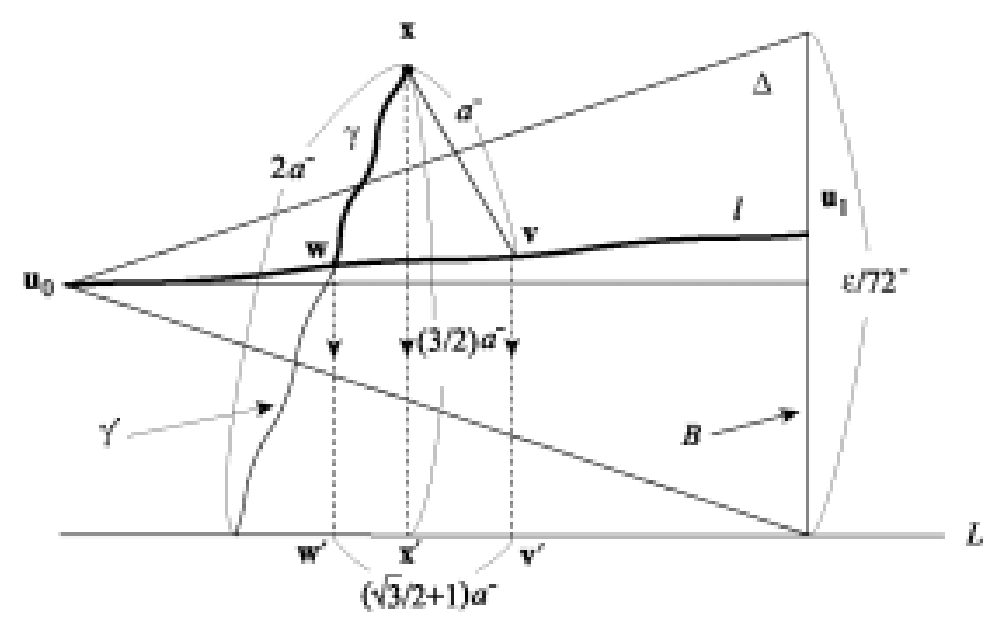

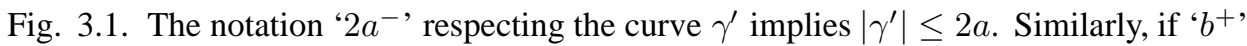
respects some curve $\delta$, then it means that $|\delta| \geq b$. This rule is applied to all the figures subsequently.

From this fact together with $T_{\mathbf{x}}(\gamma) \subset C_{\mathbf{x}}^{s}$ for any $\mathbf{x} \in \gamma \backslash\left(S \cup f^{-1}(S)\right)$, we have

$$
|\gamma| \leq\left|\gamma^{\prime}\right| \leq \frac{2}{\sqrt{3}}\left\|\mathbf{x}-\mathbf{x}^{\prime}\right\| \leq \sqrt{3} a<2 a \text {. }
$$

Note that

$$
\begin{aligned}
\left\|\mathbf{w}^{\prime}-\mathbf{v}^{\prime}\right\| & \leq\left\|\mathbf{w}^{\prime}-\mathbf{x}^{\prime}\right\|+\left\|\mathbf{x}^{\prime}-\mathbf{v}^{\prime}\right\| \leq \frac{1}{\sqrt{3}}\left\|\mathbf{x}-\mathbf{x}^{\prime}\right\|+\|\mathbf{x}-\mathbf{v}\| \\
& <\frac{1}{\sqrt{3}} \frac{3}{2} a+a=\left(\frac{\sqrt{3}}{2}+1\right) a .
\end{aligned}
$$


This implies that

$$
\left|l_{[\mathbf{w}, \mathbf{v}]}\right|<\left(1+\frac{1}{144}\right)\left(\frac{\sqrt{3}}{2}+1\right) a<2 a .
$$

This completes the proof.

Definition 3.2. Let $\left\{\mathbf{x}_{n}\right\}_{n \geq 0}$ be an $(\varepsilon, \varepsilon / 2)$-shifted $\delta$-pseudo-orbit of $f$. The pair $\left(\mathbf{x}_{n}, l_{n}\right)$ of $\mathbf{x}_{n}$ and an unstable curve $l_{n}$ in $R$ is called standard if $\left|l_{n}\right|=\varepsilon$ and $\left\|\mathbf{x}_{n}-\mathbf{v}_{n}\right\| \leq a$, where $\mathbf{v}_{n}$ is the middle point of $l_{n}$, see Figure 3.2.

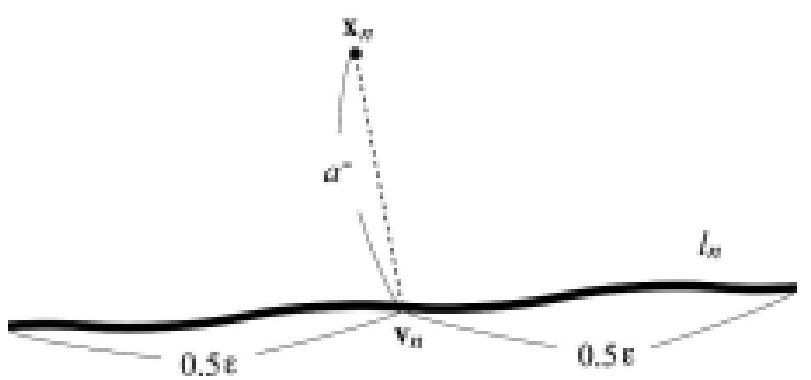

Fig. 3.2.

From the definition,

$$
\max \left\{\left\|\mathbf{x}_{n}-\mathbf{z}\right\| ; \mathbf{z} \in l_{n}\right\} \leq 0.5 \varepsilon+\frac{\varepsilon}{36}<\varepsilon
$$

\subsection{Standard pairs away from the essential singularity set}

First we consider the case that a standard pair is away from the essential singularity set $S_{1} \cup S_{2}$.

Lemma 3.3. Let $\left\{\mathbf{x}_{n}\right\}_{n \geq 0}$ be an $(\varepsilon, \varepsilon / 2)$-shifted $\delta$-pseudo-orbit for $f$ in $R$. If $\left(\mathbf{x}_{n}, l_{n}\right)$ is a standard pair with $B_{\varepsilon}\left(\mathbf{x}_{n}\right) \cap\left(S_{1} \cup S_{2}\right)=\emptyset$, then there exists an unstable curve $l_{n+1} \subset f\left(l_{n}\right)$ such that $\left(\mathbf{x}_{n+1}, l_{n+1}\right)$ is standard.

We note that the proof below works even if $B_{\varepsilon}\left(\mathbf{x}_{n}\right) \cap S_{3} \neq \emptyset$.

Proof. Suppose that $\mathbf{w}_{n}$ is the foot of $\mathbf{x}_{n}$ in $l_{n}$ and $\gamma_{n}$ is the integral curve connecting $\mathbf{x}_{n}$ with $\mathbf{w}_{n}$. By Lemma 3.1, $\left|l_{\left[\mathbf{w}_{n}, \mathbf{v}_{n}\right]}\right|<2 a$ and $\left|\gamma_{n}\right|<2 a$. The point $\mathbf{w}_{n}$ divides $l_{n}$ into two curves $l_{n}^{ \pm}$such that

$$
\left|l_{n}^{ \pm}\right| \geq 0.5 \varepsilon-\left|l_{\left[\mathbf{w}_{n}, \mathbf{v}_{n}\right]}\right|>0.5 \varepsilon-2 a>0.4 \varepsilon .
$$


See Figure 3.3(a).

By (2.1), $\left|f\left(l_{n}^{ \pm}\right)\right| \geq \sqrt{3} \cdot 0.4 \varepsilon>0.6 \varepsilon$. Thus $f\left(l_{n}\right)$ contains an unstable curve $l_{n+1}$ of length $\varepsilon$ centered at $\mathbf{v}_{n+1}=f\left(\mathbf{w}_{n}\right)$. By (3.1), $\left|f\left(\gamma_{n}\right)\right|<(1 / 8) \cdot 2 a=a / 4$. This implies that $\left\|f\left(\mathbf{x}_{n}\right)-\mathbf{v}_{n+1}\right\|=\left\|f\left(\mathbf{x}_{n}\right)-f\left(\mathbf{w}_{n}\right)\right\|<a / 4$. See Figure 3.3(b). So we have

$$
\left\|\mathbf{v}_{n+1}-\mathbf{x}_{n+1}\right\| \leq\left\|\mathbf{v}_{n+1}-f\left(\mathbf{x}_{n}\right)\right\|+\left\|f\left(\mathbf{x}_{n}\right)-\mathbf{x}_{n+1}\right\|<\frac{a}{4}+\delta<\frac{a}{3} .
$$

Thus $\left(\mathbf{x}_{n+1}, l_{n+1}\right)$ is a standard pair.

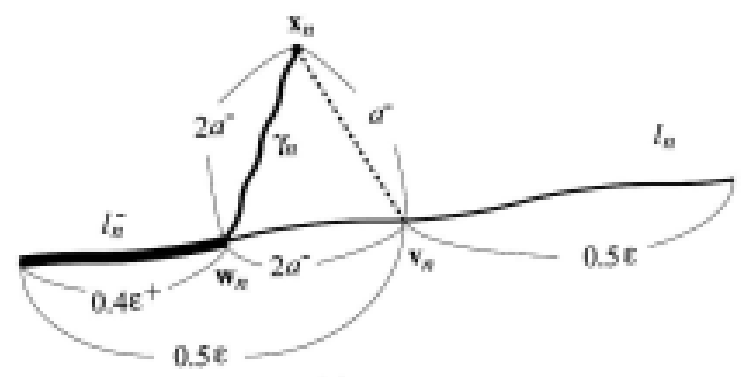

(a)

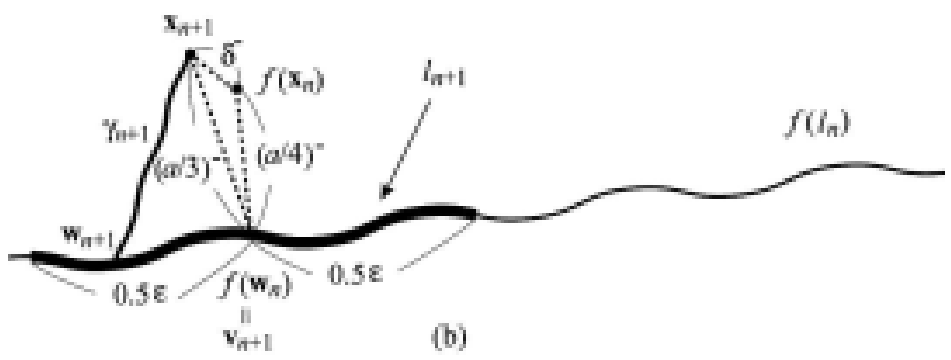

Fig. 3.3.

\subsection{Standard pairs near the essential singularity set}

In this subsection, we consider the case that the $(\varepsilon, \varepsilon / 2)$-shifted $\delta$-pseudo-orbit $\left\{\mathbf{x}_{n}\right\}_{n \geq 0}$ visits the $\varepsilon$-neighborhood of the essential singularity set $S_{1} \cup S_{2}$. Let $\left(\mathbf{x}_{n}, l_{n}\right)$ be a standard pair such that $B_{\varepsilon}\left(\mathbf{x}_{n}\right)$ meets $S_{1} \cup S_{2}$. We note that $l_{n}$ intersects $S_{1} \cup S_{2}$ at most one point. In this case, differently from the situation of Lemma 3.3, we have no guarantee to obtain an unstable curve $l_{n+1} \subset f\left(l_{n}\right)$ such that $\left(\mathbf{x}_{n+1}, l_{n+1}\right)$ is standard, see Figure 3.4 below. However, the following lemma shows that one can get a standard pair before the $(\varepsilon, \varepsilon / 2)$-shifted $\delta$-pseudo-orbit revisits the $\varepsilon$-neighborhood of $S_{1} \cup S_{2}$ again.

Lemma 3.4. Let $\left\{\mathbf{x}_{n}\right\}_{n \geq 0}$ be an $(\varepsilon, \varepsilon / 2)$-shifted $\delta$-pseudo-orbit for $f$ in $R$. If $\left(\mathbf{x}_{n}, l_{n}\right)$ is a standard pair with $B_{\varepsilon}\left(\mathbf{x}_{n}\right) \cap\left(S_{1} \cup S_{2}\right) \neq \emptyset$, then 
(i) there exists an unstable curve $l_{n+3}$ in $f^{3}\left(l_{n}\right)$ such that $\left(\mathbf{x}_{n+3}, l_{n+3}\right)$ is standard, and

(ii) $\left\|\mathbf{x}_{n+2}-\mathbf{z}\right\|<\varepsilon$ for any $\mathbf{z} \in l_{n+2}$.

Proof. First we will prove part (i) of the lemma. We may assume that $B_{\varepsilon}\left(\mathbf{x}_{n}\right) \cap S_{1} \neq \emptyset$. Note that $l_{n} \subset B_{\varepsilon}\left(\mathbf{x}_{n}\right)$. When $l_{n} \cap S_{1} \neq \emptyset$, the intersection point is denoted by $\mathbf{a}_{n}$. When $l_{n} \cap S_{1}=\emptyset$, the end point of $l_{n}$ at which $\varphi: l_{n} \rightarrow \mathbb{R}$ with $\varphi(\mathbf{x})=\pi \circ f^{2}(\mathbf{x})\left(\mathbf{x} \in l_{n}\right)$ has the maximum value is denoted by $\mathbf{a}_{n}$. Let $\gamma_{n}$ be the integral curve connecting $\mathbf{x}_{n}$ with its foot $\mathbf{w}_{n}$ in $l_{n}$. By Lemma 3.1,

$$
\left|l_{\left[\mathbf{w}_{n}, \mathbf{v}_{n}\right]}\right|<2 a, \quad\left|\gamma_{n}\right|<2 a, \quad \text { and } \quad\left\|\mathbf{x}_{n}-\mathbf{v}_{n}\right\|<a .
$$

Let $l_{n}^{-}$be the subarc of $l_{n}$ such that $\mathbf{w}_{n}$ is one of its end points and the other is the end point of $l_{n}$ other than $\mathbf{a}_{n}$. By (2.1) and (3.1),

$$
\begin{aligned}
& \left|f\left(l_{n}^{-}\right)\right|>\sqrt{3}(0.5 \varepsilon-2 a)=\sqrt{3}\left(0.5 \varepsilon-\frac{2}{36} \varepsilon\right)>0.7 \varepsilon, \\
& \left|f\left(\gamma_{n}\right)\right|<\frac{1}{8} \cdot 2 a=\frac{1}{4} a .
\end{aligned}
$$

See Figure 3.4. Thus, we get

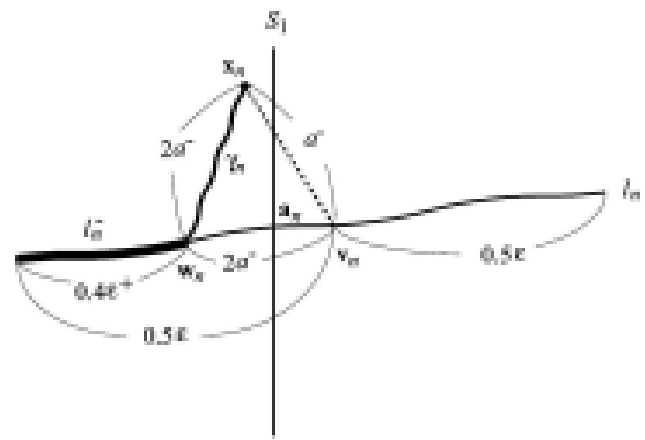

(a)

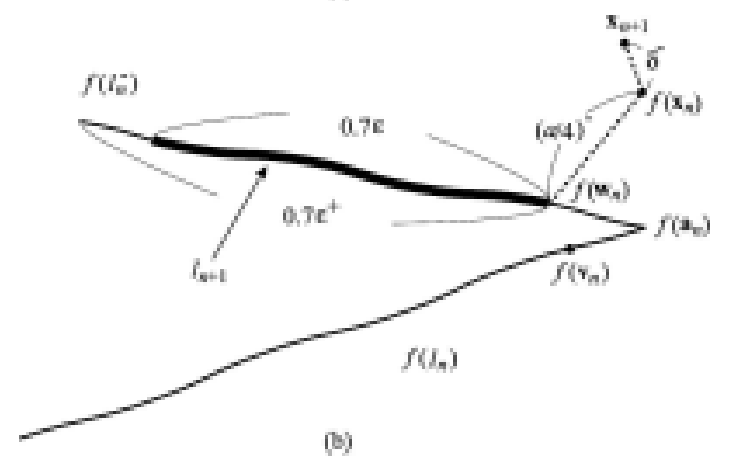

Fig. 3.4. The case of $l_{n} \cap S_{1} \neq \emptyset$. 


$$
\left\|f\left(\mathbf{w}_{n}\right)-\mathbf{x}_{n+1}\right\| \leq\left\|f\left(\mathbf{w}_{n}\right)-f\left(\mathbf{x}_{n}\right)\right\|+\left\|f\left(\mathbf{x}_{n}\right)-\mathbf{x}_{n+1}\right\| \leq \frac{1}{4} a+\delta<\frac{1}{96} \varepsilon .
$$

There exists an unstable subarc $l_{n+1}$ of $f\left(l_{n}^{-}\right)$of length $0.7 \varepsilon$ and with $\partial l_{n+1} \ni$ $f\left(\mathbf{w}_{n}\right)$. Then, we have

$$
\max \left\{\left\|\mathbf{x}_{n+1}-\mathbf{z}\right\| ; \mathbf{z} \in l_{n+1}\right\} \leq 0.7 \varepsilon+\frac{\varepsilon}{96}<\varepsilon .
$$

Note that the pair $\left(\mathbf{x}_{n+1}, l_{n+1}\right)$ is not standard. So, we have to find a standard pair in the next step.

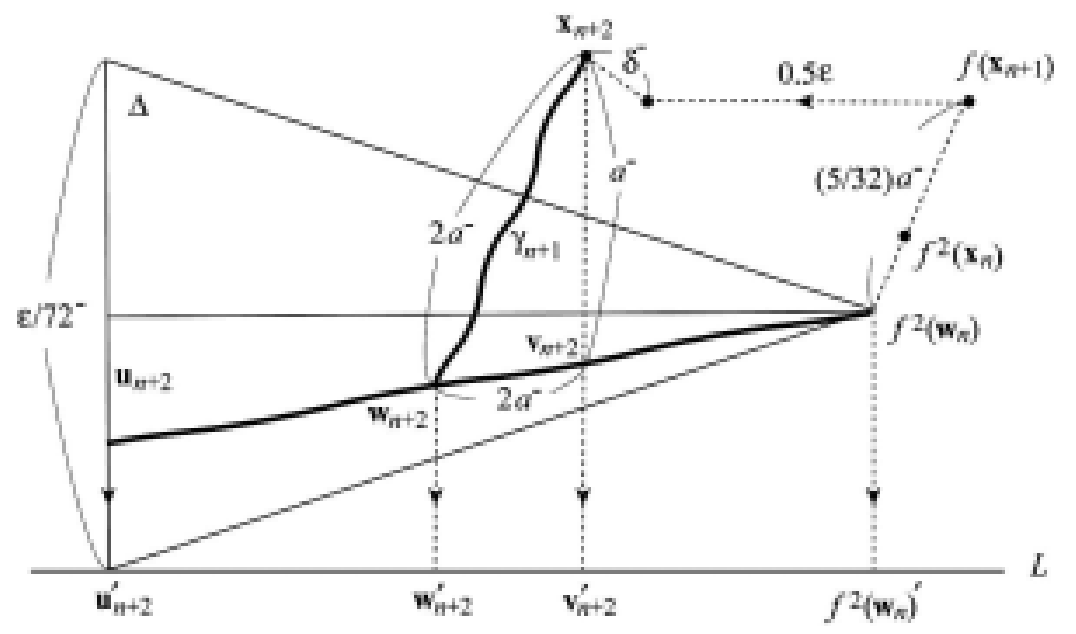

Fig. 3.5.

The image $f\left(l_{n+1}\right)$ is a curve as illustrated in Figure 3.5. From $\left|l_{n+1}\right|=0.7 \varepsilon$, $\left|f\left(l_{n+1}\right)\right| \geq \sqrt{3} \cdot 0.7 \varepsilon>1.19 \varepsilon$. Thus $f\left(l_{n+1}\right)$ contains an unstable subarc $l_{n+2}$ of length $\varepsilon$ and with $\partial l_{n+2} \ni f^{2}\left(\mathbf{w}_{n}\right)$. Set $\partial l_{n+2}=\left\{f^{2}\left(\mathbf{w}_{n}\right), \mathbf{u}_{n+2}\right\}$. By (L.2) and (3.3),

$$
\left\|f\left(\mathbf{x}_{n+1}\right)-f^{2}\left(\mathbf{x}_{n}\right)\right\| \leq M_{f} \cdot \frac{\varepsilon}{288 M_{f}}=\frac{1}{288} \varepsilon .
$$

By (3.1), $\left|f^{2}\left(\gamma_{n}\right)\right| \leq\left|\gamma_{n}\right| / 64<a / 32$. So, we have

$$
\begin{aligned}
\left\|f^{2}\left(\mathbf{w}_{n}\right)-f\left(\mathbf{x}_{n+1}\right)\right\| & \leq\left\|f^{2}\left(\mathbf{w}_{n}\right)-f^{2}\left(\mathbf{x}_{n}\right)\right\|+\left\|f^{2}\left(\mathbf{x}_{n}\right)-f\left(\mathbf{x}_{n+1}\right)\right\| \\
& \leq \frac{a}{32}+\frac{\varepsilon}{288}=\frac{a}{32}+\frac{a}{8}=\frac{5}{32} a .
\end{aligned}
$$

Consider the isosceles triangle $\Delta$ defined as in the proof of Lemma 3.1 such that the vertex is $f^{2}\left(\mathbf{w}_{n+2}\right)$ and the base $B$ contains $\mathbf{u}_{n+2}$. Let $\mathbf{v}_{n+2}$ be the intersection point of $l_{n+2}$ and the line passing through $\mathbf{x}_{n+2}$ and parallel to the $y$-axis. Note 
that $|B| \leq(2 / 144) \varepsilon=\varepsilon / 72$. As is suggested by Figure 3.5, the inequality (3.8) implies that

$$
\left\|\mathbf{x}_{n+2}-\mathbf{v}_{n+2}\right\| \leq \delta+\frac{5}{32} a+\frac{\varepsilon}{72}<a .
$$

Let $\gamma_{n+1}$ be the integral curve connecting $\mathbf{x}_{n+2}$ with its foot $\mathbf{w}_{n+2}$ in $l_{n+2}$. By Lemma 3.1,

$$
\left|\gamma_{n+1}\right|<2 a, \quad\left\|\mathbf{x}_{n+2}-\mathbf{w}_{n+2}\right\|<2 a \quad \text { and } \quad\left|l_{\left[\mathbf{w}_{n+2}, \mathbf{v}_{n+2}\right]}\right|<2 a .
$$

Let $p: \mathbb{R}^{2} \rightarrow L$ be the orthogonal projection onto a line $L$ passing through one of the end points of $B$ and parallel to the $y$-axis. For simplicity, we denote the image $p(\mathbf{x})$ of $\mathbf{x} \in \mathbb{R}^{2}$ by $\mathbf{x}^{\prime}$. The foot $\mathbf{w}_{n+2}$ divides $l_{n+2}$ into two subarcs $l_{n+2}^{ \pm}$with $l_{n+2}^{+} \ni f^{2}\left(\mathbf{w}_{n}\right)$. Then

$$
\begin{aligned}
\left|l_{n+2}^{+}\right| & \geq\left\|\mathbf{w}_{n+2}^{\prime}-f^{2}\left(\mathbf{w}_{n}\right)^{\prime}\right\| \geq\left\|\mathbf{v}_{n+2}^{\prime}-f^{2}\left(\mathbf{w}_{n}\right)^{\prime}\right\|-\left\|\mathbf{w}_{n+2}^{\prime}-\mathbf{v}_{n+2}^{\prime}\right\| \\
& >\left(0.5 \varepsilon-\delta-\frac{5}{32} a\right)-2 a>0.43 \varepsilon . \\
\left|l_{n+2}^{-}\right| & \geq\left\|\mathbf{u}_{n+2}^{\prime}-\mathbf{w}_{n+2}^{\prime}\right\| \geq\left\|\mathbf{u}_{n+2}^{\prime}-f^{2}\left(\mathbf{w}_{n}\right)^{\prime}\right\|-\left\|\mathbf{w}_{n+2}^{\prime}-f^{2}\left(\mathbf{w}_{n}\right)^{\prime}\right\| \\
& >\frac{144}{145} \varepsilon-\left(2 a+\delta+0.5 \varepsilon+\frac{5}{32} a\right)>0.42 \varepsilon .
\end{aligned}
$$

The pair $\left(\mathbf{x}_{n+2}, l_{n+2}\right)$ is still nonstandard. So, we try to get a standard pair in the next step.

Since $l_{n+2} \subset f^{2}\left(B_{\eta_{0}}\left(\mathbf{x}_{n}\right)\right), l_{n+2} \cap S=\emptyset$. Thus, by using arguments quite similar to those in Lemma 3.3, one can show that there exists an unstable subarc $l_{n+3}$ of $f\left(l_{n+2}\right)$ centered at $\mathbf{v}_{n+3}:=f\left(\mathbf{w}_{n+2}\right)$ of length $\varepsilon$ such that the pair $\left(\mathbf{x}_{n+3}, l_{n+3}\right)$ is standard. This gives the proof of part (i). Part (ii) is a simple consequence of the proof of part (i). From the above proof and $\left|l_{n+2}\right|=\varepsilon$, we get $\left|l_{n+2}^{+}\right|=$ $\left|l_{n+2}\right|-\left|l_{n+2}^{-}\right|<0.57 \varepsilon$ and $\left|l_{n+2}^{-}\right|=\left|l_{n+2}\right|-\left|l_{n+2}^{+}\right|<0.58 \varepsilon$. Then, for any $\mathbf{z} \in l_{n+2}=l_{n+2}^{+} \cup l_{n+2}^{-}$,

$$
\left\|\mathbf{x}_{n+2}-\mathbf{z}\right\| \leq\left\|\mathbf{x}_{n+2}-\mathbf{w}_{n+2}\right\|+\left\|\mathbf{w}_{n+2}-\mathbf{z}\right\|<2 a+0.6 \varepsilon<\varepsilon .
$$

This completes the proof of Lemma 3.4.

\section{Proof of Main Theorem}

Proof. ([Proof of Main Theorem]). Let $\left\{\mathbf{x}_{n}\right\}_{n \geq 0}$ be an $(\varepsilon, \varepsilon / 2)$-shifted $\delta$ pseudo-orbit of $f$ in $R$. Consider the pairs $\left(\mathbf{x}_{n}, l_{n}\right)$ which are obtained by Lemmas 3.3 and 3.4. From our construction, for any $n \geq 0, f^{-(n+1)}\left(l_{n+1}\right) \subset f^{-n}\left(l_{n}\right)$. Since each $f^{-n}\left(l_{n}\right)$ is compact, the intersection $Z=\bigcap_{n \geq 0} f^{-n}\left(l_{n}\right)$ is non-empty. By (3.5), (3.7) and (3.9), for any $\mathbf{z} \in Z$ and any integer $n \geq 0,\left\|f^{n}(\mathbf{z})-\mathbf{x}_{n}\right\|<\varepsilon$. This completes the proof. 


\title{
REFERENCES
}

1. D. V. Anosov, Geodesic flows on closed Riemann manifolds with negative curvature, Proc. Steklov Math. Inst., 90 (1967).

2. R. Bowen, $\omega$-limit sets for Axiom A diffeomorphisms, J. Diff. Eq., 18 (1975), 333-339.

3. E. M. Coven, I. Kan and J. A. Yorke, Pseudo-orbit shadowing in the family of tent maps, Trans. Amer. Math. Soc., 308 (1988), 227-241.

4. S. Kiriki, Forward limit sets of singularities for the Lozi family, Hokkaido Math. Jour, 33 (2004), 491-510.

5. S. Kiriki and T. Soma, Parameter-shifted shadowing property of Lozi maps, Dyn. Syst., 22(3) (2007), 351-363.

6. S. Kiriki and T. Soma, Parameter-shifted shadowing property for geometric Lorenz attractors, Trans. Amer. Math. Soc., 357 (2005), 1325-1339.

7. Z. Liu, H. Xie, Z. Zhou and Q. Lu, The strange attractor of the Lozi mapping, Internat. J. Bifur. Chaos Appl. Sci. Engrg., 2(4) (1992), 831-839.

8. R. Lozi, Un attracteur étrange du type attracteur de Hénon, J. Physics (Paris), 39 (1978), 69-77.

9. M. Misiurewicz, Strange attractors for the Lozi mappings, Nonlinear dynamics (InternationalConference, New York, 1979), Annals of the New York Academy of Sciences, Vol. 357, New York Academy of Sciences, New York, 1980, pp. 348-358,

10. H. E. Nusse and J. A. Yorke, Is every approximate trajectory of some process near an exact trajectory of a near process? Comm. Math. Phys., 114 (1988), 363-379.

11. L.-S. Young, A Bowen-Ruelle measure for certain piecewise hyperbolic maps, Trans. Amer. Math. Soc., 287 (1985), 41-48.

\author{
Akio Sakurai \\ Department of Mathematics and Information Sciences, \\ Tokyo Metropolitan University, \\ Minami-Ohsawa 1-1, \\ Hachioji, Tokyo 192-0397, \\ Japan \\ E-mail: a.sakurai.0828@gmail.com
}

